\title{
The analysis of a conveyor-serviced production station
}

\author{
W.M. NAWIJN \\ Twente University of Technology, Enschede, Netherlands \\ and \\ R. DE JONGE \\ Dr. Neher Laboratory, P.T.T., Leidschendam, Netherlands
}

Received May 1979

Revised October 1979

This paper gives a queuing analysis of a conveyor-serviced production station using a state-dependent sequential range policy for unloading units from the conveyor into a reserve. The stationary distribution of the number of units in the reserve and the expectel delay per unit processed are derived for a Poisson arrival precess. The form of the optimal unloading policy, which ninimizes the expected delay, will be established.

\section{Introduction}

Conveyor theory has been principally concerned with two distinctive types of systems: the automatically unloaded, multi-server systems and the workerloaded--unloaded systems. (For a survey of Conveyor Theory, see White and Muth [6].) In the latter type of system the unloading of units from the conveyor and the processing of units at the work-station are executed by the same person, consequently production is delayed during unloading activity. In a number of articles probabilistic models have been developed for the single worker-unloading production station, see e.g. [1-5]. Research is focused on the development of efficient unloading policies which should minimize unloading delay and consequently increase the productivity of the work station.

In this paper a new unloading policy is introduced and an analysis of this policy is given. This policy is more general then the state-dependent policy investigated by Matsui and Shingu [4], both policies being generalisations of the 'Sequential Range Policy', introduced by Beightler and Crisp [1].

(c) North-Holland Publishing Company

Evropean Journal of Operational Research 6 (1981) 67-74

\subsection{Description of the model}

Units are transported by means of an irreversible, continuous belt conveyor, moving with constant speed. The units are unloaded from the conveyor by an operator, at a production station situated at some fixed point along the conveyor and stored in a storage, referred to as reserve. After the unloading, the same operator takes one unit from the reserve and performs the necessary operations on the unit. Processed units leave the system. The cycle of the operator's productive activity can be divided into two distinct, sequential operations, i.e., the unloading operation and the actual productive operation. The length of the unloading operation will be referred to as 'delay per unit processed'. All units arriving during the processing of a unit are lost.

The unloading of units, using the newly proposed strategy, proceeds as follows: upon terminating the processing of a unit, the operator turns to the conveyor and inspects a range of $c_{i}$ time units, i.e., that part of the conveyor that will pass the station within a time $c_{i}$, if the number of units in the reservebank equals $i$. If the range contains one or more units the operator delays and unloads the first unit to arrive and stores it in the reserve after which a new range of length $c_{i+1}$ is inspected, beginning this range at the arrival of the unit just obtained. This procedure continues until either the reserve is filled or a range is inspected that does not contain a unit. The operator then returns to work.

If, upon terminating a processing, the operator finds the reserve empty, he delays until a unit arrives, i.e., $c_{0}=\infty$, after which inspection starts with a range $c_{1}$.

For a graphical representation of a typical productive cycle see Fig. 1.

The following assumptions are made:

The arrivals of units at the unloading point constitutes a stationary Poisson process with arrivalrate $\lambda$.

All handling and walkingtimes are negligible.

The reserve has a finite capacity $K+1$, i.e., $C_{K+1}=0$.

The operator's sight along the conveyor, measured in time units, is at least equal to the maximal range. 


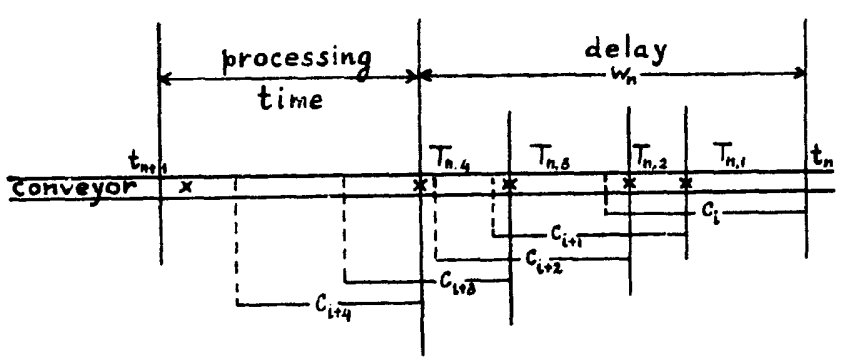

Fig. 1. A Eypical productive cycle.

Moreover, it will be supposed that the processingtimes are always greater than or equal to $\max \left(c_{1}, \ldots\right.$, $\left.c_{K}\right)$. This assumption guarantees that the time interval between the end of a processing and the subsequent arrival of a unit is negative exponentially distributed with mean $1 / \lambda$.

\section{The content of the reserve}

Let the successive completions of processing occur at the moments $t_{n}, n \geqslant 0$. By $x_{n}$, we denote the number of units in the reserve at the time $t_{n}+0$. By $u\left(x_{n}\right)$ we denote the number of units unloaded between $t_{n}$ and $t_{n+1}$, given the value of $x_{n}$. Clearly

$x_{n+1}=x_{n}+u\left(x_{n}\right)-1, \quad n \geqslant 0$.

It follows from the assumptions made that the sequence $\left\{x_{n}, n \geqslant 0\right\}$ forms a Markov chain with finite state space $S=\{0,1, \ldots, K\}$.

Denoting by $q_{i j}=\operatorname{Pr}\left\{x_{n+1}=j \mid x_{n}=i\right\}$ the onestep transition probabilities it can be verified that

$$
\begin{aligned}
& q_{i j}=\mathrm{e}^{-\lambda c_{j+1}} \prod_{k=i}^{j}\left(1-\mathrm{e}^{-\lambda c_{k}}\right) \\
& \quad \text { for } 1 \leqslant i \leqslant K, \quad i-1 \leqslant j \leqslant K-1, \\
&=\prod_{k=i}^{K}\left(1-\mathrm{e}^{-\lambda c_{k}}\right) \\
& \quad \text { for } 1 \leqslant i \leqslant K, \quad j=K \\
&=\mathrm{e}^{-\lambda c_{j+1}} \prod_{k=1}^{j}\left(1-\mathrm{e}^{\left.-\lambda c_{k}\right)}\right. \\
& \quad \text { for } i=0, \quad 0 \leqslant j \leqslant K-1 \\
&=0 \quad \text { for } i=0, \quad j=K \\
& \quad \text { elsewhere, }
\end{aligned}
$$

in which the convention is used that $\Pi_{1}^{0}(\cdot)=1$.

From (2.2) it is seen that the chain is time-homogeneous, aperiodic and irreducible. So the chain $\left\{x_{n}, n \geqslant 0\right\}$, having a finite state space, is ergodic. Consequently, it possesses a unique stationary distribution $\left\{v_{i}, i=0,1, \ldots, K\right\}$, which is the solution of the equations

$v_{j}=\sum_{i=0}^{K} v_{i} q_{i j}, \quad j=0,1, \ldots, K, \quad$ and

$\sum_{i=0}^{K} v_{j}=1$.

On substituting (2.2) in (2.3) it can be shown that the set of equations (2.3) is equivalent to

$$
\begin{gathered}
v_{1}=\mathrm{e}^{\lambda c_{1}}\left(1-\mathrm{e}^{-\lambda c_{1}}\right) v_{0}, \\
v_{j+1}=\mathrm{e}^{\lambda c_{j+1}} v_{j}-\mathrm{e}^{\lambda c_{j}\left(1-\mathrm{e}^{-\lambda c_{j}}\right) v_{j-1},} \\
\quad j=1,2, \ldots, K-1,
\end{gathered}
$$

$\sum_{i=0}^{K} v_{i}=1$.

It can be readily verified that the solution is given by

$v_{j}=v_{0} \prod_{i=1}^{j}\left(\mathrm{e}^{\lambda c_{i}}-1\right), \quad j=1,2, \ldots, K$,

$v_{0}=1 \mid\left\{1+\sum_{j=1}^{K} \prod_{i=1}^{j}\left(\mathrm{e}^{\lambda} c_{i}-1\right)\right\}$.

Note that in case $c_{i}=c, i=1,2, \ldots, K$ the model of Crisp [1], [2] is obtained, except that here the arrival process is Poisson instead of Bernoulli. In this case the set of equations (2.4) describes a classical random walk with two reflecting barriers.

From (2.1) it follows, noting that $E\left\{x_{n}\right\}$ tends to a finite limit, that

$\lim _{n \rightarrow \infty} E\left\{u\left(x_{n}\right)\right\}=1$,

i.e., the expected number of unloaded units during a productive cycle equals one in the stationary state, as indeed it must.

\section{The mathematical expectation of the delay per unit processed}

Let $w_{n}$ be defined as the delay of the operator in removing units from the conveyor between $t_{n}$ and 
$t_{n+1}$, i.e., the length of the unloading operation during the $n$th productive cycle. The random variable $w_{n}$ is composed of the successive interarrival times during the unloading period, denoted by $T_{n, i}, i=$ $1,2, \ldots$.

In Fig. 1 a realisation of a productive cycle, starting with $i$ units in the reserve, is shown.

By assumption it follows for $k=1,2, \ldots, j-i+1$ and $0 \leqslant i \leqslant j \leqslant K$ that

$$
\begin{array}{rlrl}
\operatorname{Pr}\left\{T_{n, k} \leqslant t \mid x_{n}=i, x_{n+1}=j\right\} & \\
& =\frac{1-\mathrm{e}^{-\lambda t}}{1-\mathrm{e}^{-\lambda c_{i+k-1}},}, & 0 \leqslant t \leqslant c_{i+k-1}, \\
& =1 & t>c_{i+k-1},
\end{array}
$$

and

$$
\begin{aligned}
& E\left\{T_{n, k} \mid x_{n}=i, x_{n+1}=j\right\} \\
& \quad=\frac{1}{\lambda}-c_{i+k-1} \frac{\mathrm{e}^{-\lambda c_{i+k-1}}}{1-\mathrm{e}^{-\lambda c_{i+k-1}}} .
\end{aligned}
$$

By definition, we have

$$
\begin{aligned}
& E\left\{w_{n} \mid x_{n}=i, x_{n+1}=j\right\} \\
& =\sum_{k=1}^{j-i+1} E\left\{T_{n, k} \mid x_{n}=i, x_{n+1}=j\right\} \text { for } 0 \leqslant i \leqslant j \leqslant K .
\end{aligned}
$$

It then follows from (3.2) that

$$
\begin{aligned}
& E\left\{w_{n} \mid x_{n}=i, x_{n+1}=j\right\} \\
& =\sum_{k=i}^{j}\left[\frac{1}{\lambda}-c_{k} \frac{\mathrm{e}^{-\lambda c_{k}}}{1-\mathrm{e}^{-\lambda c_{k}}}\right], \quad 0 \leqslant i \leqslant j \leqslant K,
\end{aligned}
$$

which is obviously zero for $j<i$.

Consider the equality

$$
\begin{aligned}
E\left\{w_{n}\right\}=\sum_{i=0}^{K} \sum_{j=0}^{K} & \operatorname{Pr}\left\{x_{n}=i\right\} q_{i j} \\
& \times E\left\{w_{n} \mid x_{n}=i, x_{n+1}=j\right\},
\end{aligned}
$$

on substituting in this the expressions from (2.2) and (3.3), we obtain after rearranging terms

$$
\begin{gathered}
E\left\{w_{n}\right\}=\frac{1}{\lambda} \sum_{i=0}^{K} \sum_{j=0}^{K}(j-i+1) \operatorname{Pr}\left(x_{n}=i\right) q_{i j} \\
-\sum_{k=0}^{K} c_{k} \frac{\mathrm{e}^{-\lambda c_{k}}}{1-\mathrm{e}^{-\lambda c_{k}}} \\
\times \sum_{i=0}^{k} \sum_{j=k}^{K} \operatorname{Pr}\left(x_{n}=i\right) q_{i j} .
\end{gathered}
$$

Hence, from (2.1), (2.2) and noting that $c_{0}=\infty$,

$$
\begin{aligned}
E\left\{w_{n}\right\}= & \frac{1}{\lambda} E\left\{u\left(x_{n}\right)\right\}-\sum_{k=1}^{K} c_{k} \frac{\mathrm{e}^{-\lambda c_{k}}}{1 \cdots \mathrm{e}^{-\lambda c_{k}}} \\
& \times\left[\sum_{i=0}^{k} \operatorname{Pr}\left(x_{n}=i\right)-\sum_{i=0}^{k-1} \operatorname{Pr}\left(x_{n+1}=i\right)\right] \\
& \text { for } n \geqslant 1 .
\end{aligned}
$$

It will be observed that $E\left\{u\left(x_{n}\right)\right\}$ equals the expected number of unloaded units during the $n$th productive cycle. Let $w$ denote the unloading delay per cycle in the stationary state, then we have, taking the limit for $n \rightarrow \infty$, (cf. 2.6),

$E\{w\}=\frac{1}{\lambda}-\sum_{k=1}^{K} c_{k} v_{k} \frac{\mathrm{e}^{-\lambda c_{k}}}{1-\mathrm{e}^{-\lambda c_{k}}}$,

or (cf. 2.5) equivalently

$E\{w\}=\frac{1}{\lambda}-\sum_{k=1}^{K} c_{k} v_{k-1}$,

where the stationary probabilities $\left\{v_{i}, i=0,1, \ldots, K\right\}$ are given in (2.5).

For the special case $c_{1}=c_{2}=\cdots=c_{K}=c$ one finds

$E\{w\}=\frac{1}{\lambda}-\frac{c \mathrm{e}^{-\lambda c}}{1-\mathrm{e}^{-\lambda c}}\left(1 \cdots v_{0}\right)$,

in agreement with a result of Beightler and Crisp [1] adapted to a Poisson arrival process.

\section{The optimal unloading policy}

A policy which minimizes the average delay per cycle will be called an optimal policy. In this section it will be shown that there exists a unique optimal policy $\left(c_{1}^{*}, \ldots, c_{K}^{*}\right)$ satisfying the following property $c_{1}^{*}>r_{2}^{*}>\cdots>c_{K}^{*}$, i.e., the policy is monotone with respect to the state variable. To this end a number of lemmas will be proved. Writing $n$ for $K$, the average delay can be written as

$E\{w\}=\frac{1}{\lambda}-F_{n}\left(c_{1}, \ldots, c_{n}\right)$,

where the function $F_{n}$ is defined as, (cf. (2.5) and (3.7)),

$F_{n}\left(c_{1}, \ldots, c_{n}\right)=\frac{G_{n}\left(c_{1}, \ldots, c_{n}\right)}{H_{n}\left(c_{1}, \ldots, c_{n}\right)}$, 
in which

$G_{n}\left(c_{1}, \ldots, c_{n}\right)=\sum_{i=1}^{n} c_{i} \prod_{j=1}^{i-1}\left(\mathrm{e}^{\lambda c_{j}}-1\right)$,

and

$H_{n}\left(c_{1}, \ldots, c_{n}\right)=\sum_{i=1}^{n+1} \prod_{j=1}^{i-1}\left(\mathrm{e}^{\lambda c_{j}}-1\right)$.

Obviously, minimizing $E\{w\}$ is equivalent to maximizing $F_{n z}$. It should be noted that the optimization problem can be formulated as a Markovian decision process. Here, instead, we treat the problem as a classical optimization problem.

Lemma 1. The function $F_{n}\left(c_{1}, \ldots, c_{n}\right)$ takes on its maximum in the open set $D_{n}\left\{\left(c_{1}, \ldots, c_{n}\right) \mid 0<c_{i}<\infty\right.$, $i=1,2, \ldots, n\}$.

Proof. Let the function $F_{n}\left(c_{1}, \ldots, c_{n}\right)$ with $c_{i} \geqslant 0$, $i=1,2, \ldots, n$, be transformed into a function $\widetilde{F}_{n}\left(x_{1}, \ldots, x_{n}\right)$, with $0 \leqslant x_{i} \leqslant 1, i=1,2, \ldots, n$, using the transformation $x_{i}=\exp \left(-\lambda c_{i}\right)$. The following properties of the function $\widetilde{F}_{n}, n \geqslant 2$, are easily verified:

$$
\text { (i) } \begin{aligned}
\tilde{F}_{n} & \left(x_{1}, \ldots, x_{i-1}, 1, x_{i+1}, \ldots, x_{n}\right) \\
& =\tilde{F}_{i-1}\left(x_{1}, \ldots, x_{i-1}\right), \quad i=2, \ldots, n,
\end{aligned}
$$

anid

$$
\text { (ii) } \begin{aligned}
\tilde{F}_{n} & \left(x_{1}, \ldots, x_{k}, \ldots, x_{n}\right) \\
& \rightarrow F_{n-k}\left(x_{k+1}, \ldots, x_{n}\right) \\
& \text { if } x_{k} \downarrow 0, \quad k=1,2, \ldots, n-1,
\end{aligned}
$$

and

$$
\begin{aligned}
& \tilde{F}_{n}\left(x_{1}, \ldots, x_{n-1}, 0\right)=0 . \\
& \frac{\partial \tilde{F}_{n}}{\partial x_{n}}=-\prod_{i=1}^{n-1} \frac{\left(1-x_{i}\right)}{x_{i}} \\
& \quad \times\left(\frac{1}{\lambda}-\tilde{F}_{n-1}\left(x_{1}, \ldots, x_{n-1}\right)\right\} / H_{n-1}, \\
& \quad \text { for } x_{n} \uparrow 1 .
\end{aligned}
$$

(iv) $\tilde{F}_{n}\left(x_{1}, \ldots, x_{n}\right)$, considered as a function of $x_{1}$ only, takes on its maximum in the open interval $(0,1)$ for all values of $x_{i}, i=2, \ldots, n$, with $0 \leqslant x_{i} \leqslant 1$, $i=2, \ldots, n$.

$$
\text { (v) } \tilde{F}_{n}\left(x_{1}, \ldots, x_{n}\right)<\frac{1}{\lambda} \text {. }
$$

This property follows from (4.1) by noting that $w>0$ almost su ely, if $0<\lambda<\infty$.

The function $\widetilde{F}_{n}$ is a continuous function on a closed and bounded set, therefore it takes on its global maximum on this set, according to a wellknown theorem of Weierstrass. Let us denote by $\bar{x}_{i}(n), i=1,2, \ldots, n$ the values which maximize $\bar{F}_{n}$.

From (4.2) we have $\tilde{F}_{1}\left(x_{1}\right)=-1 / \lambda x_{1} \ln x_{1}$ and it is readily verified that this function has its maximum at a point of $\tilde{D}_{1}=\left\{x_{1} \mid 0<x_{1}<1\right\}$. Now consider the function $\widetilde{F}_{2}\left(x_{1}, x_{2}\right)$. From Property (iv) it follows that $0<\bar{x}_{1}(2)<1$. Moreover from (ii) it follows that $\bar{x}_{2}(2)>0$. On combining the Properties (iii) and (v) and noting that $0<x_{1}<1$ it is seen that $\partial \widetilde{F}_{2} / \partial x_{2}<0$, for $x_{2} \uparrow 1$, consequently $0<\bar{x}_{2}(2)<1$.

On taking $x_{1}=\bar{x}_{1}(1)$ and $x_{2}=1$ in (iii) and using Property (i) it follows that $\widetilde{F}_{1}^{*}<\widetilde{F}_{2}^{*}$, where $\widetilde{F}_{i}^{*}=$ $\widetilde{F}_{i}\left(\bar{x}_{1}(i), \bar{x}_{2}(i), \ldots, \bar{x}_{i}(i)\right), i=1,2, \ldots, n$. Suppose that the functions $\widetilde{F}_{j}\left(x_{1}, \ldots, x_{j}\right), j=1,2, \ldots, k(<n)$, take on their global maximum $\widetilde{F}_{i}^{*}$ in the open set $\widetilde{D}_{j}=$ $\left\{\left(x_{1}, \ldots, x_{j}\right) \mid 0<x_{i}<1, i=1,2, \ldots, j\right\}$ and moreover that $\widetilde{F}_{1}^{*}<\widetilde{F}_{2}^{*}<\cdots<\widetilde{F}_{k}^{*}$.

From this assumption we will now prove that the same is true for $j=1,2, \ldots, k+1$. If one or more variables of $\widetilde{F}_{k+1}$ equal zero or one, then the function $\widetilde{F}_{k+1}$ is reduced to a function $\widetilde{F}_{j}\left(x_{1}, \ldots, x_{j}\right)$, with $j \leqslant k$, according to the Properties (i) and (ii). From the assumption made it follows that in case $\widetilde{F}_{k+1}$ is optimal $j$ will not be less than $k$. The only cases to be considered in which a variable is on its boundary are $x_{1}=0,0<x_{i}<1, i=2, \ldots, k+1$ and $0<x_{i}<1$, $i=1,2, \ldots, k, x_{k+1}=1$. The first case cannot give a maximum taking into account Property (iv). On combining the Properties (iii) and (v) one finds that $\partial \widetilde{F}_{k+1} / \partial x_{k+1}<0$ for $x_{k+1}=1$ so that the second case can equally not give a maximum, which finally implies that $\widetilde{F}_{k+1}$ must take on its global maximum in the open set $\widetilde{D}_{k+1}$. Because $\widetilde{F}_{k}^{*}=\widetilde{F}_{k+1}\left(\bar{x}_{1}(k), \ldots, \bar{x}_{k}(k), 1\right)$ it also follows from the Properties (iii) and (v) that $\tilde{F}_{k}^{*}<\widetilde{F}_{k+1}^{*}$.

Consequently if the assumption made for the functions $\widetilde{F}_{j}, j=1,2, \ldots, k$ holds, it also holds for $1 \leqslant j \leqslant$ $k+1$. Noting that the assumption holds for $k=2$ it follows by mathematical induction that the functions $\widetilde{F}_{j}, j=1,2, \ldots, n$, have their global maximum in the sets $\check{D}_{j}, j=1,2, \ldots, n$. Now it should be observed that $F_{n}$ has a maximum in $\left(\overline{c_{1}}, \ldots, \overline{c_{n}}\right)$, with $0<\overline{c_{i}}<\infty$, $i=1,2, \ldots, n$ if and onily if $\widetilde{F}_{n}$ has a maximum in $\left(\bar{x}_{1}, \ldots, \bar{x}_{n}\right)$ with $0<\bar{x}_{i}<1$ and $\bar{x}_{i}=\exp \left(-\lambda \bar{c}_{i}\right)$, $i=1,2, \ldots, n$, due to the monotony of the transformation. Moreover, from $\bar{x}_{i}(n)>0$ we have $\overline{c_{i}}(n)<\infty$. 
So $F_{n}$ is optimal in $D_{n}=\left\{\left(c_{1}, \ldots, c_{n}\right) \mid 0<c_{i}<\infty\right.$, $i=1,2, \ldots, n\}$.

The maximum of the function $F$, dropping the subscript, satisfies the necessary conditions $\partial F / \partial c_{i}=0$, $i=1,2, \ldots, n$.

The solutions of these equations are called the stationary points of $F$. Noting that $F$ has continuous first order derivatives on $D_{n}$, Lemma 1 implies that there exists at least one stationary point.

Lemma 2. The stationary points of the function $F$ in the set $D_{n}$ satisfy the equations

$\lambda c_{i}=\lambda c_{i+1}+\mathrm{e}^{-\lambda c_{i+1}}-\mathrm{e}^{-\lambda c_{i-1}}, \quad i=1,2, \ldots, n$,

with $c_{0}=\infty$ and $c_{n+1}=0$.

Proof. The stationary points of $F$ satisfy the equations

$H \frac{\partial G}{\partial c_{i}}-G \frac{\partial H}{\partial c_{i}}=0, \quad i=1,2, \ldots, n$,

because $H>0$ for all $c_{i} \geqslant 0$. This leads to

$$
\begin{array}{r}
H\left\{1+\lambda \mathrm{e}^{\lambda c_{i}} \sum_{i=i+1}^{n} c_{j} \prod_{k=i+1}^{j-1}\left(\exp \left(\lambda c_{k}\right)-1\right)\right\} \\
-\lambda G \mathrm{e}^{\lambda c_{i}} \sum_{j=i+1}^{n} \prod_{k=i+1}^{j-1}\left(\exp \left(\lambda c_{k}\right)-1\right)=0,
\end{array}
$$

considering only solutions in $D_{n}$.

On taking $i=n$ in (4.4) gives

$H-\lambda \mathrm{e}^{\lambda c_{n} G}=0$.

Considering $i=n-1$ in (4.4), we have

$\left(1+\lambda c_{n} \mathrm{e}^{\lambda c_{n-1}}\right) H-\lambda G \mathrm{e}^{\lambda c_{n}+\lambda c_{n-1}}=0$.

Substituting (4.5) in (4.6) yields

$\lambda c_{n}=1-\mathrm{e}^{-\lambda c_{n-1}}$.

Again, considering (4.4) for $i=n-2$ and using (4.5) and (4.6) leads, after simplification, to

$\lambda c_{n-1}=1-\mathrm{e}^{-\lambda c_{n-1}}-\mathrm{e}^{-\lambda c_{n-2}}+\mathrm{e}^{-\lambda c_{n}}$.

In proceeding this way for $i=n-3, n-4, \ldots, 1$ one finds the following set of equations

$$
\begin{aligned}
& H-\lambda G \mathrm{e}^{\lambda c_{n}}=0, \\
& \lambda c_{i}=1-\mathrm{e}^{-\lambda c_{i}}-\mathrm{e}^{-\lambda c_{i}-1}+\mathrm{e}^{-\lambda c_{n}}, \\
& \quad i=2,3, \ldots, n .
\end{aligned}
$$

Now it can be deduced that the first equation is equivalent to

$\lambda c_{1}=1-\mathrm{e}^{-\lambda c_{1}}+\mathrm{e}^{-\lambda c_{n}}$,

by substituting the equations for $i=2,3, \ldots, n$ in (4.9) into the first. Then from (4.9) and (4.10) it is seen that

$\lambda c_{i}=\lambda c_{i+1}+\mathrm{e}^{-\lambda c_{i+1}}-\mathrm{e}^{-\lambda c_{i-1}}, \quad i=1,2, \ldots, n$,

with $c_{0}=\infty$ and $c_{n+1}=0$, which proves the lemma.

Lemma 3. Any finite positive solution $\left(c_{1}, \ldots, c_{n}\right)$ of the equations (4.3) satisfies $c_{1}>c_{2}>\cdots>c_{n}>0$.

Proof. The first equation in (4.3) reads

$\lambda c_{1}=\lambda c_{2}+\mathrm{e}^{-\lambda c_{2}}$,

hence $c_{1}>c_{2}$.

Adding the equations for $i=1,2, \ldots, j-1, j>i$, in (4.3) gives

$\lambda c_{1}=\lambda c_{j}-\mathrm{e}^{-\lambda c_{1}}+\mathrm{e}^{-\lambda c_{j}}+\mathrm{e}^{-\lambda c_{j-1}}$.

Suppose $c_{1}>c_{k}$, for $k=2,3, \ldots, j \ldots 1$, then from (4.11) we have $c_{1}>c_{j}$; thus, $c_{1}>c_{2}$ being true, it follows by mathematical induction that $c_{1}>c_{k}$, $k=2, \ldots, n$. Now suppose that

$c_{k}>c_{j}$, for $j=k+1, \ldots, n$

and $k=1,2, \ldots, i<n$.

From (cf. (4.3))

$\lambda c_{i+1}=\lambda c_{i+2}+\mathrm{e}^{-\lambda c_{i+2}}-\mathrm{e}^{-\lambda c_{i}}$,

it is easily seen that $c_{i+1}>c_{i+2}$, because, by assumption $c_{i}>c_{j+2}$. Moreover, suppose $c_{i+1}>c_{m}$ holds for $m=i+2, \ldots, r$. From (4.11) we have

$\lambda c_{i+1}=\lambda c_{r+1}-\mathrm{e}^{-\lambda \lambda_{i}}+\mathrm{e}^{-\lambda c_{r+1}}-\mathrm{e}^{-\lambda c_{i+1}}+\mathrm{e}^{-\lambda c_{r}}$.

Then it is easily seen that $c_{i+1}>c_{r+1}$, having assumed that $c_{i}>c_{r+1}$ and $c_{i+1}>c_{r}$, consequently if assumption (4.12) holds up to $i$, it holds for $i+1$ also. Now, noting that (4.12) is true for $i=1$, it is true for $i=1,2, \ldots, n$ by induction.

We have seen that the function $F$ possesses at least one stationary point. We will prove now that this is the only stationary point in $D_{n}$.

Lemma 4. The finite positive solution of the equations (4.3) is unique.

Proof. Suppose that the equations (4.3) admit two bounded solutions $\left(c_{1}, \ldots, c_{n}\right)$ and $\left(d_{1}, \ldots, d_{n}\right)$. Then 
both solutions satisfy Lemma 3 . Assume that $c_{1}>d_{1}$. Then from (4.3) it follows that

$\mathrm{e}^{-\lambda c_{n}}-\mathrm{e}^{-\lambda d_{n}}=\left(\lambda c_{1}+\mathrm{e}^{-\lambda c_{1}}\right)-\left(\lambda d_{1}+\mathrm{e}^{-\lambda d_{1}}\right)>0$,

noting that $c_{1}>d_{1}>0$ and that the function between the brackets is strictly increasing. Consequently, we have $c_{n}<d_{n}$.

The following equality is easily established

$$
\begin{aligned}
& \left(\lambda c_{j+1}+\mathrm{e}^{-\lambda c_{j+1}}\right)-\left(\lambda d_{j+1}+\mathrm{e}^{-\lambda d_{j+1}}\right) \\
& \quad=\left(\lambda c_{1}+\mathrm{e}^{-\lambda c_{1}}\right)-\left(\lambda d_{1}+\mathrm{e}^{-\lambda d_{1}}\right)+\left(\mathrm{e}^{-\lambda d_{j}}-\mathrm{e}^{-\lambda c_{j}}\right) .
\end{aligned}
$$

From this it is readily verified by an inductive argument that if $c_{1}>d_{1}$ then $c_{i}>d_{i}$ for $i=1,2, \ldots, n$, which apparently leads to a contradiction. Hence $c_{1}>d_{1}$ is impossible. Starting with the assumption $c_{1}<d_{1}$, again a contradiction is found, consequently one must have $c_{1}=d_{1}$. Then, using (4.3) it is seen that $c_{i}=d_{i}, i=1,2, \ldots, n$.

Lemma 5. The optimal vaiue of $E\{w\}$ satisfies

$\lambda E\{w\}=1-\mathrm{e}^{-\lambda c_{n}^{*}}$.

Proof. This immediately follows from the relations (4.1), (4.2) and (4.5).

Combining the results of the five lemmas we finally have

\section{Theorem 1. There exists a unique optimal policy} $\left(c_{1}^{*} \cdots c_{n}^{*}\right)$ minimizing the average delay per unit processed, with the following properties:

(i) The ranges $c_{i}^{*}, i=1,2, \ldots, n$ are the unique positive bounded solution of the equations

$$
\begin{aligned}
\lambda c_{i}^{*} & =\lambda c_{i+1}^{*}+\mathrm{e}^{-\lambda c_{i+1}^{*}}-\mathrm{e}^{-\lambda c_{i-1}^{*}}, \\
i & =1,2, \ldots, n,
\end{aligned}
$$

with $c_{0}^{*}=\infty$ and $c_{n+1}^{*}=0$

(ii) $c_{1}^{*}>c_{2}^{*}>\cdots>c_{n}^{*}>c_{n+1}^{*}=0$.

(iii) $\lambda E\{w\}=1-\mathrm{e}^{-\lambda c_{n}^{*}} \quad$ for $c_{i}=c_{i}^{*}, i=1, \ldots, n$.

Up to now no consideration has been given to the influence of the reserve capacity on the unloading policy. Let $\left(c_{1}(n), \ldots, c_{n}(n)\right)$ denote the optimal unloading policy for $K=n, n \geqslant 1$. The influence of the reservecapacity on the unloading policy is given by the following:

Theorem 2. The optimal unloading ranges $c_{i}(n)$, $i=1,2, \ldots, n$ are monotone increasing functions of the reservecapacity $n$, i.e., $c_{i}(n-1)<c_{i}(n), i=$ $1,2, \ldots, n$.
Proof. The ranges $c_{i}(n), i=1,2, \ldots, n$ and $c_{i}(n-1)$, $i=1, \ldots, n$ satisfy the equation (of. (4.3))

$$
\begin{aligned}
& {\left[\lambda c_{j+1}(n-1)+\exp \left\{-\lambda c_{j+1}(n-1)\right\}\right]} \\
& \quad-\left[\lambda c_{j+1}(n)+\exp \left\{-\lambda c_{j+1}(n)\right\}\right] \\
& =\left[\lambda c_{1}(n-1)+\exp \left\{-\lambda c_{1}(n-1)\right\}\right] \\
& \quad-\left[\lambda c_{1}(n)+\exp \left\{-\lambda c_{1}(n)\right\}\right] \\
& \quad+\exp \left\{-\lambda c_{j}(n)\right\}-\exp \left\{-\lambda c_{j}(n-1)\right\}, \\
& j=1,2, \ldots, n .
\end{aligned}
$$

Suppose $c_{1}(n-1) \geqslant c_{1}(n)$, analogous to the proof of Lemma 4, it follows by mathematical induction that $c_{i}(n-1) \geqslant c_{i}(n)$ for $i=1,2, \ldots, n$. However, noting that $c_{n}(n-1)=0$, this implies $c_{n}(n) \leqslant 0$, which, according to Theorem 1 , is not true. Therefore we must have $c_{1}(n-1)<c_{1}(n)$. Again, using mathematical induction it then follows that $c_{i}(n-1)<$ $c_{i}(n), i=1,2, \ldots, n$.

\section{Numerical considerations}

In order to obtain the solution of the equations (4.3) the following successive approximation procedure was used:

$$
\begin{aligned}
\lambda c_{1}^{(n+1)}= & \lambda c_{2}^{(n)}+\exp \left\{-\lambda c_{2}^{(n)}\right\}, \\
\lambda c_{i}^{(n+1)}= & \lambda c_{i+1}^{(n)}+\exp \left\{-\lambda c_{i+1}^{(n)}\right\} \\
& -\exp \left\{-\lambda c_{i-1}^{(n+1)}\right\}, \quad i=2, \ldots, K-1, \\
\lambda c_{K}^{(n+1)}= & 1-\exp \left\{-\lambda c_{K-1}^{(n)}\right\},
\end{aligned}
$$

starting with $c_{i}^{(1)}=0, i=1,2, \ldots, K$.

Table 1 gives the values of the optimal ranges for several values of the reserve capacity. Note the monotonicity properties in accordance with Theorems 1 and 2.

In Table 2 an illustrative comparison is given between the policies of Crisp [2], Matsui and Shingu [4] and our policy. In the second column the optimal value of $\lambda c$ for Crisp's policy is given. The third and fourth columns show the largest and smallest optimal ranges of our policy. The last three columns give the optimal mean delay for the three policies (for the. policy of Matsui and Shingu the only two optimal values which could be found are given).

The policy presented here is the best of the three, which will be evident keeping in mind that Crisp's policy corresponds to the case $c_{1}=c_{2}=\cdots=c_{K}$, and 
Table 1

Optimal ranges

\begin{tabular}{rllllllllll}
\hline$K$ & $\lambda c_{1}$ & $\lambda c_{2}$ & $\lambda c_{3}$ & $\lambda c_{4}$ & $\lambda c_{5}$ & $\lambda c_{6}$ & $\lambda c_{7}$ & $\lambda c_{8}$ & $\lambda c_{9}$ & $\lambda c_{10}$ \\
\hline 2 & 1.20 & 0.70 & & & & & & & & \\
3 & 1.28 & 0.86 & 0.58 & & & & & & \\
4 & 1.33 & 0.95 & 0.72 & 0.52 & & & & & \\
6 & 1.39 & 1.03 & 0.85 & 0.72 & 0.60 & 0.45 & & & \\
8 & 1.41 & 1.07 & 0.91 & 0.80 & 0.72 & 0.64 & 0.55 & 0.42 & & 0.40 \\
10 & 1.42 & 1.09 & 0.94 & 0.84 & 0.77 & 0.71 & 0.66 & 0.60 & 0.52 & 0.40 \\
\hline
\end{tabular}

Table 2

Comparison with other policies

\begin{tabular}{rllllll}
\hline$K$ & $\lambda c$ & $c_{1}^{*}$ & $c_{K}^{*}$ & Crisp & M\&S & $\lambda E\{w\}$ \\
\hline 2 & 0.90 & 1.20 & 0.70 & 0.5177 & & 0.5021 \\
3 & 0.85 & 1.28 & 0.58 & 0.4625 & 0.4521 & 0.4388 \\
4 & 0.85 & 1.33 & 0.52 & 0.4305 & & 0.4026 \\
6 & 0.80 & 1.39 & 0.45 & 0.3939 & & 0.3644 \\
7 & 0.80 & 1.40 & 0.44 & 0.3832 & 0.3629 & 0.3534 \\
8 & 0.75 & 1.41 & 0.42 & 0.3746 & & 0.3453 \\
10 & 0.75 & 1.42 & 0.40 & 0.3616 & & 0.3345 \\
15 & 0.75 & 1.44 & 0.39 & 0.3447 & & 0.3213 \\
20 & 0.75 & 1.45 & 0.38 & 0.3371 & & 0.3156 \\
30 & 0.70 & 1.45 & 0.37 & 0.3275 & & 0.3109 \\
50 & 0.70 & 1.46 & 0.37 & 0.3189 & & 0.3078 \\
\hline
\end{tabular}

the fact that Matsui and Shingu use the same range during the produrtion cycle, which depends only on the number of usables in the reserve just after the termination of the service.

The relative gain over Crisp's policy in using our policy is at most $8 \%$. In case Morris' single unit policy is used, for which $c_{1}=c_{2}=\cdots=c_{K}=\infty$, the mean delay equals $\lambda^{-1}$.

Taking this policy as level of reference the relative gain over Crisp's policy is only $3 \%$.

\section{Conclusion}

Beightler and Crisp [1] and Crisp [2], introduced the so called 'Sequential Range Policy', (SRP). This policy is a special case of the policy considered here and can be obtained by putting $c_{1}=c_{3}=\cdots=c_{K}=c$. In his thesis Crisp suggested to generalize SRP in that the unloading ranges should be dependent on the current number of units in the reserve. Independently, Matsui and Shingu [4], investigated a state-dependent sequential range policy, which strongly resembles the policy considered here. The difference is that instead of setting the size of the range every moment an unloading have taken place, they use the same range during one production cycle, the size of which depends only on the number of units in the reserve just after the termination of the processing. From a mathematical point of view it seems that our policy is a more natural generalisation of the SRP. Com paring the state dependent policies with SRP, see also [4], shows that the gain over SRP is marginal, taking the case $K=0$ as level of reference. Moreover the state dependent policies are more difficult to implement, which is a practical drawback. To overcome this drawback one could introduce simplified policies, using a smaller number of ranges, see [4].

\section{References}

[1] C.S. Beightler and R.M. Crisp, Jr., A discrate-time queuing analysis of conveyor-serviced production stations, Operations Res. 16 (5) (1968) 986-1001.

[2] R.M. Crisp, Jr., An analysis of conveyor-serviced production stations, Ph.D. Dissertation, University of Texas, Austin, TX (1967). 
[3] M. Matsui and J. Fukuta, A queuing analysis of conveyorserviced production station with general unit-arrival, $J$. Operations Res. Soc. Japan, $18(3,4)(1975) 211-227$.

[4] M. Matsui and T. Shingu, A queuing analysis of conveycrserviced production station and the optimal range strategy, AIIE Trans. 10 (1) (1978) 89-99.
[5] L.L. Reis, J.J. Brennan and R.M. Crisp, A Markovian analy sis for delay at conveyor-serviced production stations, Internat. J. Prod. Res. 5 (3) (1967) 201-211.

[6] J.A. White and E.J. Muth, Conveyor theory: A survey, Proc. Sogesci Conference on Analysis of Material Handling Systemis, Antwerpen, Belgium, 1977. 